\title{
SUMMARY ON ORIENTATIONS OF ROTATION AXES
}

\author{
CARL D. VESELY \\ University of Arizona
}

To evaluate the precision of the previously determined coordinates of the rotation axes (table I) we should review the methods (originally developed for 433 Eros) and logic of the various authors. Table II contains pole coordinates of Eros and the sources of these data. A critical summary of the work will enable us to make some conclusions concerning the poles presented.

\section{EARLY RESEARCH ON THE POLE OF EROS}

There is general agreement that greatest rotational amplitude is observed when an asteroid is viewed equatorially, and we can detect three approaches to the determination of the Eros pole: the micrometer position angles observed by van den Bos and Finsen (1931); the graphic presentations used by Watson (1937), Stobbe (1940), and Rosenhagen (1932); and the mathematical model developed by Krug and Schrutka-Rechtenstamm (1936). These initial attempts yielded only approximate values, but the approximations were sometimes refined by analytical methods.

\section{Micrometer Measurements of Position Angles}

Eros is the only asteroid to have directly observed micrometer measurements of the position angles of the projection of its long axis. Van den Bos and Finsen (1931) found the position angle rotating over $360^{\circ}$ in $5^{\mathrm{h}} 17^{\mathrm{m}}$ and a separation of "about 0 ". 18 ." The precision of the measurements of the position angle may be $\pm 5^{\circ}$ (Van Biesbroeck, personal communication).

In $1931, \mathrm{~W}$. Zessewitsche $(1932,1937)$ graphically determined the equator of Eros from the observations of van den Bos and Finsen (1931). He determined an average position angle of the line of intersection of the projection of the long axis of Eros with the projection plane perpendicular to the line of sight at a given time and assumed the pole to be this position angle plus $90^{\circ}$. Zessewitsche calculated a value for the inclination of the equator of Eros to the projection plane at that time and determined the pole coordinates. The pole determination enabled him to calculate the Erocentric right ascension 


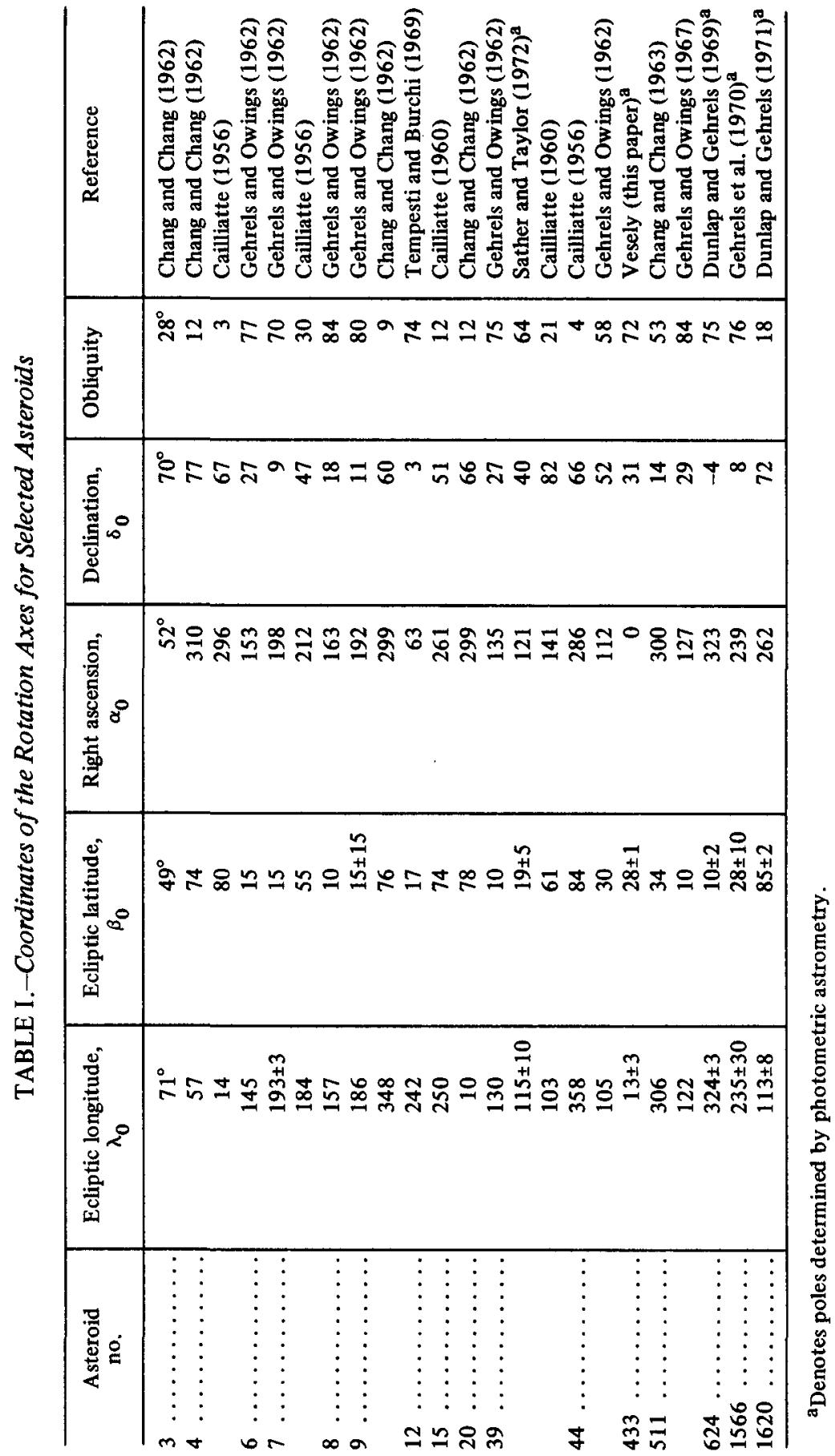


TABLE II.-Pole Coordinates of Asteroid 433 Eros

\begin{tabular}{l|r|r|r|r}
\hline \multicolumn{1}{c|}{ Reference } & $\lambda_{0}$ & $\beta_{0}$ & $\alpha_{0}$ & $\delta_{0}$ \\
\hline Zessewitsche (1937) & $29^{\circ}$ & $22^{\circ}$ & $18^{\circ}$ & $31^{\circ}$ \\
Rosenhagen (1932) & 4 & 45 & 342 & 42 \\
Watson (1937) & 349 & 62 & 316 & 51 \\
Krug and Schrutka- & & & & \\
$\quad$ Rechtenstamm (1936) & 2 & 53 & 333 & 48 \\
Stobbe (1940) & 9 & 38 & 350 & 38 \\
Beyer (1953) & 353 & 13 & 349 & 9 \\
Cailliatte (1956) & 10 & 46 & 345 & 45 \\
Vesely (this paper) & 13 & 28 & 0 & 31 \\
\hline
\end{tabular}

and declination $d$ of the Earth. Plotting the observed amplitude $A$ against $d$, Zessewitsche derived the following empirical relationship:

$$
A=1^{\mathrm{m}} \cdot 50-0^{\mathrm{m}} \cdot 0275|d|
$$

where $1.50 \mathrm{mag}$ is the maximum observed amplitude. We refer to this as an amplitude-aspect relationship.

Zessewitsche's method relies on a large number of transformation equations. The value for the inclination of the equator is an important parameter in several of them. Zessewitsche admitted a lack of precision in its calculation.

M. Huruhata (1940) misinterpreted Zessewitsche's pole value and his work must be viewed with suspicion.

\section{Graphic Pole Determinations}

F. Watson (1937) plotted the observed amplitude as a function of the ecliptic coordinates of Eros during an opposition. Assuming that the greatest amplitude was observed when Earth was in the equatorial plane of Eros, Watson used a composite of the curves of oppositions from 1893 to 1935 to secure the coordinates of the nodes of the equatorial plane of Eros and its inclination, from which he determined the pole.

Watson used published amplitudes from various oppositions, combining photometric and photographic data without indicating the probable error of the observed magnitudes and included data regarded as imprecise by Stobbe (1940). Checking the original published lightcurves, I found variations between different observers on the same night to be as high as $\pm 0.3 \mathrm{mag}$, and I completely reject his pole.

J. Rosenhagen obtained an approximate pole from a plot of the amplitude against the Earth's ecliptic coordinates. The refinement will be discussed under "Mathematical Models for Pole Determinations."

J. Stobbe (1940) used Zessewitsche's amplitude-aspect relationship to determine $d_{B}$, the Erocentric latitude of Earth from the observed amplitude. 
With various assumed pole positions, he calculated a series of $d_{R}$ values ( $B=$ beobachtet; $R=$ rechnet). Using the $d_{B}$ values as normal points, curves of the $d_{R}$ values for the various assumed pole values were plotted for the dates of observation. The results were indeterminate, so he used another relationship as follows.

By using a period calculated from the previously approximated pole, Stobbe found $\left(a_{\min } \cdot f\right)_{B}$, the observed variation in the time of arrival of the minimum due to change in phase. Using the assumed pole positions, he determined $\left(a_{\min } \cdot f\right)_{R}$, the calculated variation. The resulting $B-R$ plot, along with the $d_{R}$ plot, enabled Stobbe to elect the best assumed pole from pole curves. To determine possible agreement between his possible poles for the 1930-31 opposition and van den Bos and Finsen's (1931) position angles, Stobbe plotted his pole coordinates as points and drew the great circle corresponding to the position angle of the pole. He presumed the intersection of the points and the great circle to be the pole of Eros. The result agreed with one of his values, but not the one he felt best represented the pole of Eros for the opposition 1930-31. Stobbe indicated the disagreement was possibly due to irregularity of the figure or flexure along the long axis and claimed his findings vindicated the often skeptically received (Stobbe, 1940) observations of van den Bos and Finsen (1931).

Although Stobbe selected one pole as most satisfying, we see he has as many poles as he has oppositions. He claimed the pole is not fixed. On the contrary, I believe the slope of an amplitude-aspect plot determined for observations at a certain phase angle and obliquity will be valid for that opposition only and will yield a different pole for another opposition unless the conditions are the same. Stobbe rejected obviously unsure observational data, but the ones he accepted may not be accurate. The inability to secure a single pole may indicate systematic error of the method.

\section{Mathematical Models for Pole Determinations}

J. Rosenhagen (1932) showed that his graphically approximated pole (see above) must be refined in terms of Eros' shape. He assumed an elongated body rotating about the short axis, similar to a Poincaré body or symmetrical egg figure, with a brightness proportional to the projected area when viewed equatorially. To yield an Eros maximum amplitude of $1.50 \mathrm{mag}$ required an axial ratio of $a: b=4.00$ and an eccentricity of $e=0.97$. Rosenhagen devised an amplitude-aspect relationship based on this model. Starting with the approximate pole, he made differential corrections until he determined the pole yielding the aspect that best conformed to the requirements of the model.

Rosenhagen found that his pole gave the right amplitude for the observations of 1930-31, but would give a maximum amplitude of only 1.14 mag for the earlier oppositions (1901-1903). He blamed systematic deviations of the data due to precession, deformation, and spotting of the asteroid. 
Rosenhagen's pole may be challenged for more obvious reasons. He tried to intercompare amplitudes among oppositions whose observational data produced a range of phase coefficients from 0.011 to $0.039 \mathrm{mag} / \mathrm{deg}$ and included data that Watson (1937) said was not comparable because of uncertainties in the magnitudes of the comparison stars. Rosenhagen's pole generates little confidence.

W. Krug and G. Schrutka-Rechtenstamm (1936) proposed to determine the brightness of a three-axis ellipsoid model of Eros at full phase while obeying Lambert's law, using only photometric observational data having both amplitude and absolute magnitude reduced to an average opposition. They related absolute brightness to the aspect angle, which was determined from Rosenhagen's pole. A least-squares solution gave a corrected pole. Krug's new pole met the brightness conditions required by the model, but did not permit sufficient maximum amplitude.

Because Krug and Schrutka-Rechtenstamm's pole would not permit maximum amplitude, and they used data from observers common to Rosenhagen, criticized previously, this pole, too, must be considered very doubtful.

F. E. Roach and L. G. Stoddard (1938) revised the work of Krug and Schrutka-Rechtenstamm. They assumed Krug's pole, but related the brightness ratio to the maximum amplitude, omitted observations with no variation, and gave no weight to absolute magnitude. Their least-squares solution allowed for a maximum amplitude of $1.50 \mathrm{mag}$ for Eros. Thus, using old, imprecise photometric data and their own, single photoelectric lightcurve, Stoddard and Roach perhaps improved a model, but shed no further light on Eros' pole.

\section{MORE RECENT POLE DETERMINATIONS}

M. Beyer (1953) used Stobbe's (1940) method for his determination of the pole of Eros. Although his observational data are more precise, the pole seems to be unreasonably low.

Cailliatte (1956) used the geocentric coordinates of an asteroid for two observations of the maximum amplitude to determine the longitude of the node and the inclination of the equator of the asteroid. The pole thus determined was used to calculate $D$, the asterocentric declination of Earth. Cailliatte then plotted an amplitude-aspect relation that he refined using various models. He used the refined amplitude-aspect relationship to correct the original pole. In a later publication (1960) he corrected two earlier poles. Cailliatte's amplitude-dependent method required larger than observed maximum amplitudes for some asteroids (e.g., for 39 Laetitia: $0.68 \mathrm{mag}$ calculated, 0.54 mag observed) and yields generally small obliquities.

Y. C. Chang and C. S. Chang $(1962,1963)$ determined a number of poles using an amplitude-aspect relationship. They used a single reduced observation and the asteroid's phase coefficient as the factor by which the amplitude varies 
with $D$, citing Cailliatte (1956) as the source. Actually, Cailliatte indicated this was a "restrictive hypothesis," somewhat better than no method at all. The Chang poles have no value.

P. Tempesti and R. Burchi (1969) also made use of an amplitude-aspect relationship:

$$
A_{i}=A_{0}-C|d|
$$

where $A_{i}$ is one of 12 observed amplitudes, $A_{0}$ is an assumed maximum (unknown), $C$ is a constant (unknown), and $d$ is the asterocentric declination of Earth. They used each $A_{i}$ and increasing values of $C$ with each assumed $A_{0}$. A least-squares solution analyzing the relative minima of residuals indicated $A_{0}=1.50 \mathrm{mag}$ and $C=0.0146 \mathrm{mag}^{-\mathrm{deg}^{-1}}$ yielded minimal standard error. They transformed the value received for $d$ into pole coordinates. Tempesti and Burchi stated the error may be large because of the small range of amplitudes. Greater faults appear evident. There is no observational justification of a maximum amplitude of $1.50 \mathrm{mag}$. Also, a partial lightcurve (41/2 hr) of April 6, 1970, gives an amplitude of 0.07 mag at a time when, according to Tempesti, a nearly equatorial view was anticipated.

The early poles of T. Gehrels and D. Owings (1962) were determined using an amplitude-aspect relationship:

$$
a=A|\sin \gamma|
$$

where $a$ is the observed amplitude, $A$ the greatest possible amplitude, and $\gamma$ the angle between the direction of observation and the axis of rotation. $A \sin \gamma$ master curves were made for different values of $A$ and $\beta_{0}$ plotted as a function of $\left(\lambda-\lambda_{0}\right) \cdot \beta=0$ was assumed; and when only two observations were available, it was assumed $\beta_{0}=8^{\circ}$. The master curves were superposed on longitude plots of $a_{1}, a_{2}$, and the visual absolute magnitude. A weighted average was given for the determined longitude $\lambda_{0}$, giving half-weight to the absolute magnitude and to poor determinations. The latitude of the pole was determined from the quality of fit to the observations by the different sets of master curves. Gehrels claims little precision for the latitudes and no determination of a sign. The observational data are good and the phase angles were usually small. The pole longitudes are more precise, as they do not depend strongly on the assumed amplitude-aspect function.

Recognizing the unreliability of the amplitude-aspect relationship, Gehrels (1967) developed the photometric astrometry method described by R. Taylor ${ }^{1}$ and determined the pole of 4 Vesta in 1967. An error in cycle correction discovered later causes us to lack confidence in this determination. We believe the pole is within $\pm 10^{\circ}$ of the published coordinates.

${ }^{1}$ See p. 128. 
The poles of asteroids 39 (Sather and Taylor, 1972), 624 (Dunlap and Gehrels, 1969), 1566 (Gehrels et al., 1970), and 1620 (Dunlap and Gehrels, 1971) are determined from photometric astrometry. My present photometric astrometry determination of the pole of Eros was done utilizing the observational data of Beyer (1953) for the opposition of 1951-52. This is a preliminary value from the one opposition only.

\section{CRITICAL SUMMARY AND CONCLUSIONS}

The precision of Zessewitsche's pole is dependent on the accuracy of the micrometer measurements of the position angle and the value for the inclination of the equator. Zessewitsche recognized the lack of precision in the latter. The poles of Watson, Rosenhagen, and Krug depend on the precision of values determined for the absolute brightness and amplitude obtained in many oppositions. This precision is poor. Stobbe and Beyer used more precise data within oppositions, but had to choose a "best" pole from among several possible poles.

With increased observational precision in recent years, we might expect more reliable pole determinations. We see from table I, however, that little agreement exists. Discounting the apparently incorrect poles of Chang and Tempesti, we still see great disagreement between the poles of Cailliatte and Gehrels.

It may be concluded, then, that we must challenge the fundamental validity of the amplitude-aspect relationship upon which great doubt has already been cast by J. L. Dunlap ${ }^{2}$ and seek a more reliable way to determine a precise pole position. Photometric astrometry shows great promise, and we are currently engaged in a program using and further improving that method.

\section{ACKNOWLEDGMENTS}

This program is supported by the National Aeronautics and Space Administration.

\section{REFERENCES}

Beyer, M. 1953, Der Lichtwechsel und die Lage der Rotationsachse des Planeten 433 Eros während der Opposition 1951-52. Astron. Nachr. 281, 121-130.

Bos, W. H. van den, and Finsen, W. S. 1931, Physical Observations of Eros. Astron. Nachr. 241, 329-334.

Cailliatte, C. 1956, Contribution à l'Étude des Astéroïdes Variables. Bull. Astron. Paris 20, 283-341.

Cailliatte, C. 1960, Contribution à l'Étude des Astéroïdes Variables (Suite). Publ. Observ. Lyon 6(1), pp. 259-272.

Chang, Y. C., and Chang, C. S. 1962, Photometric Investigations of Seven Variable Asteroids. Acta Astron. Sinica 10, 101-111.

\footnotetext{
${ }^{2}$ See p. 151.
} 
Chang, Y. C., and Chang, C. S. 1963, Photometric Observations of Variable Asteroids, II. Acta Astron. Sinica 11, 139-149.

Dunlap, J. L., and Gehrels, T. 1969, Lightcurves of a Trojan Asteroid. Astron. J. 74, 796-803.

Dunlap, J. L., and Gehrels, T. 1971, Minor Planets and Related Objects. VIII. Astron. J. 76, in press.

Gehrels, T. 1967, The Rotation of Vesta. Astron. J. 72, 929-938.

Gehrels, T., and Owings, D. 1962, Photometric Studies of Asteroids. IX. Additional Light-Curves. Astrophys. J. 135, 906-924.

Gehrels, T., Roemer, E., Taylor, R. C., and Zellner, B. H. 1970, Asteroid (1566) Icarus. Astron. J. 75, 186-195.

Huruhata, M. 1940, The Rotation Period of Eros. Harvard Col. Observ. Circ. 442, pp. 1-6.

Krug, W., and Schrutka-Rechtenstamm, G. 1936, Untersuchungen über Gestalt und Grösse des Planetoiden Eros. Z. Astrophys. 13, 1-12.

Roach, F. E., and Stoddard, L. G. 1938, A Photoelectric Light-Curve of Eros. Astrophys. J. 88, 305-312.

Rosenhagen, J. 1932, Einige Bemerkungen zur Helligkeit und zum Lichtwechsel des Planeten Eros. Mitt. Wien. Sternw. 1(2), 45-52.

Sather, R. E., and Taylor, R. C. 1972, Minor Planets and Related Objects. IX. Astron. J., in preparation.

Stobbe, J. 1940, Der Lichtwechsel des Eros. Astron. Nachr. 270, 1-24.

Tempesti, P., and Burchi, R. 1969, A Photometric Research on the Minor Planet 12 Victoria. Mem. Soc. Astron. Ital. 40, 415-432.

Watson, F. 1937, The Physical Nature of Eros. Harvard Col. Observ. Circ. 419, pp. 1-14.

Zessewitsche, W. 1932, Die Bestimmung der Winkelelemente der inneren Bewegung von Eros. Astron. Nachr. 246, 441-450.

Zessewitsche, W. 1937, On the Rotation of Eros. Observatory 60, 289-292. 